\title{
Dalla penuria di guerra alla società del benessere. Il caso della Francia. Intervista a Jean Pierre Williot, Università di Tours
} ISSN: 2282-4979 DOI: $10.12977 /$ ereview113

Cosa accade in Francia durante la Seconda Guerra Mondiale sul fronte dell'alimentazione? Come avviene il passaggio dalla guerra al dopoguerra? Nel rispondere a queste domande Jean Pierre Williot pone i termini di una comparazione fra Francia e Italia. Una scelta che, nell'evidenziare elementi di contiguità o difformità, stabilisce un punto di partenza per un'interpretazione aperta alla dimensione europea.

What happened in France during World War II in the field of feeding? How was the transition from war to post war? Jean Pierre Williot gives answer to these questions through a comparison between France and Italy. It is a choice which highlights both analogies and differences with an European point of view.

\section{Introduzione}

Jean Pierre Williot è professore di Storia contemporanea all'Università François Rabelais di Tours, direttore del Laboratoire d'études sur l'alimentation e vicepresidente nel consiglio Scientifico dell'Institut européen d'histoire et des cultures alimentaires. I suoi temi di ricerca riguardano la storia dei prodotti alimentari e dell' approvvigionamento urbano, il rapporto fra alimentazione e mobilità, i processi di trasmissione delle culture alimentari e la storia delle tecniche di cottura. Nell'intervista, raccolta per il dossier nel corso dell'estate 2016, Williot spiega come in Francia, negli anni della Seconda guerra mondiale e a seguito di una profonda destabilizzazione del mercato alimentare, lo stato inaugurò una politica di 
razionamento che istituzionalizzò una sorta di "penuria organizzata", utile a garantire un equilibrio fra ciò che veniva prodotto e quanto poteva essere distribuito. Dopo il 1942, con l'occupazione dell'intero territorio nazionale, la situazione peggiorò anche a causa del prelevamento di quote di prodotto da parte delle forze occupanti, condizione alla quale le campagne resistettero più efficacemente, mentre le città subirono i danni più vistosi a causa di un circuito di approvvigionamento ormai inesistente, aprendo la strada al mercato nero.

Nel biennio 1944-1945 si registrarono denutrizione o insufficienza alimentare per buona parte della popolazione, con effetti demografici e sulla salute pubblica all'indomani del conflitto. E tuttavia, di contro, alcune categorie di popolazione continuarono ad alimentarsi sufficientemente anche grazie alla possibilità di accedere a circuiti di approvvigionamento paralleli a quelli ufficiali. Affrontare la penuria costrinse a nuove abitudini - di consumo, di preparazione dei cibi - per le quali era necessario acquisire competenze. Ricettari di guerra e trasmissioni radiofoniche furono vettori di una pedagogia utile all'adattamento, sulla base di valutazioni di ordine salutistico e nutrizionale. Paradossalmente le condizioni di guerra costituirono un'occasione per ripensare a stili di vita e di alimentazione, e se le mentalità non furono ancora pronte alla trasformazione, si crearono i presupposti per evoluzioni future.

Una situazione di normalità venne ristabilita all'inizio degli anni Cinquanta, ma molto più a lungo permase un disgusto per prodotti e alimenti che erano stati, durante il conflitto, gli unici possibili. Fu in tale contesto che l'industria alimentare rispose a un'esigenza di novità e sicurezza, in una società sempre più urbanizzata e aperta al consumo. Si trattò di un'abbondanza liberatoria: mangiare molto assumeva il significato di liberazione dalla miseria. In questo clima di mutamento il modello gastronomico della ristorazione francese - riemerso negli anni Cinquanta grazie al desiderio di recuperare lo standard dell'anteguerra - entrò in crisi nel corso degli anni Settanta per la sua inadeguatezza di fronte alle nuove esigenze di una società in profonda trasformazione. Quando sulla scena irruppero le raffinatezze di una nouvelle cuisine ancora marcatamente nazionale e francese, $\mathrm{i}$ processi di decolonizzazione e i fenomeni migratori ampliarono la prospettiva, fino ad aprire alle cucine d'Oltremare. 


\section{Intervista}

In Francia quali sono gli effetti sull'alimentazione portati dalla Seconda guerra mondiale?

\section{Q https://www.youtube.com/watch?v=sHwfPPFhxI0}

C'è un crollo del mercato interno nelle ultime fasi del conflitto? E qual è la situazione negli anni immediatamente successivi alla fine della guerra?

Q https:/www.youtube.com/watch?v=YHrWhATeSTQ

In Italia ci sono libri di ricette che aiutano ad affrontare la particolare situazione della penuria di guerra. Esiste qualcosa di simile in Francia?

Attps://www.youtube.com/watch?v=MM8X0C6Uxdk

È possibile comparare le due guerre mondiali per quanto concerne il tema dell'alimentazione? Quali aspetti emergono?

O https://www.youtube.com/watch?v=TsB1C1yGWp8

Si può sostenere che i problemi dell'approvvigionamento provocati dalla guerra abbiano in qualche modo stimolato l'evoluzione dell'industria alimentare nei decenni successivi?

O https://www.youtube.com/watch?v=tahfceckr1Y

In Italia il richiamo alla memoria della fame di guerra ha un ruolo fondamentale per il suo superamento. Si può dire sia così anche in Francia?

Q https://www.youtube.com/watch?v=0IDQJkZrWzM

Allo scoppio della Seconda guerra mondiale il modello di Escoffier identifica la cucina francese nel mondo. Come irrompe il conflitto nella traduzione e riproposizione di questo mito gastronomico?

$\mathbf{O}$ https://www.youtube.com/watch?v=h214d-HEZk

Si può parlare di una contaminazione fra la cucina francese e le cucine dei paesi colonizzati dalla Francia?

O https://www.youtube.com/watch?v=79q58 keMls 